\title{
Revisiting the area of the open cluster Stock $16^{\star}, \star \star$
}

\author{
R. A. Vázquez ${ }^{1, \star \star \star}$, G. L. Baume ${ }^{1,2, \star \star \star}$, C. Feinstein ${ }^{1, \star \star \star}$, J. A. Nuñez ${ }^{1, \star \star \star}$, and M. M. Vergne $e^{1, \star \star \star}$ \\ ${ }^{1}$ Facultad de Ciencias Astronómicas y Geofísicas UNLP, IALP-CONICET, Paseo del Bosque s/n, 1900, \\ La Plata, Argentina \\ e-mail: rvazquez@fcaglp.fcaglp.unlp.edu.ar \\ 2 Dipartimento di Astronomía, Università di Padova, Vicolo Osservatorio 2, 35122 Padova, Italy
}

Received 15 April 2004 /Accepted 10 August 2004

\begin{abstract}
Deep CCD $U B V(R I)_{\mathrm{C}}$ photometry has been carried out in the area of the open cluster Stock 16 . The different photometric diagrams indicate the presence of three superimposed stellar groups, all composed of early type stars. The first of them, the cluster Stock 16, suffers from slightly variable reddening with a mean color excess $\left\langle E_{B-V}\right\rangle=0.51$. The second group, reddened by $\left\langle E_{B-V}\right\rangle=0.84$, includes the Wolf-Rayet star WR 50 (a WC7+OB), and the third one, with a mean $\left\langle E_{B-V}\right\rangle=1.18$, also includes another Wolf-Rayet star, WR 51 (a WN4+OB?). Our results confirm the distance and reddening already stated for Stock 16 but include new members. Concerning the other two highly reddened groups, we show that the first one - located at a distance of $3600 \mathrm{pc}$ - is probably related to an anonymous OB association behind Cen OB1 also at $3600 \mathrm{pc}$ already mentioned in the literature; the other seems to be a distant $\mathrm{OB}$ association - at more than $9000 \mathrm{pc}$ - which may belong to the Scutum-Crux spiral arm. In these two groups we find evidence that the absorption law, $A_{V} / E_{B-V}=R$ amounts to 4.0 approximately. The ages of the different star populations in the zone range from $5 \times 10^{6} \mathrm{yr}$ to $6.4 \times 10^{6} \mathrm{yr}$ for Stock $16,8 \times 10^{6}$ for the second most reddened group and $5 \times 10^{6}$ for the probable association in Scutum-Crux. A determination of the IMF slope of Stock 16 was also carried out for stars in the mass range $1.8<M<14 M_{\odot}$, giving a normal value $x=1.3$.
\end{abstract}

Key words. Galaxy: open clusters and associations: individual: WR 50 - stars: luminosity function, mass function Galaxy: open clusters and associations: individual: WR 51 - stars: imaging

\section{Introduction}

The very young open cluster Stock 16 lies close to the east border of the Coal Sack in Crux $(l=306.11, b=+0.14)$ where it appears projected against the HII region RCW 75 (Rodgers et al. 1960), probably excited by the UV radiation field from the O7.5 V ((f)) star HD 115455 (Walborn 1973). A few photometric studies of the brightest members of Stock 16 have been carried out in the past, e.g. Lyngå (1970), Crampton (1971) and Lundström \& Stenholm (1984). Later on, Turner (1985; hereafter T85) performed a detailed analysis in Stock 16 and the surrounding area by means of $U B V$ photometry for 33 stars finding a color excess $E_{B-V}=0.49$ and a distance of $1.90 \pm$ $0.08 \mathrm{kpc}$ which coincides with the distance estimation, also quoted by T85, of Cen OBI and Cen Rl $(1.88 \pm 0.24 \mathrm{kpc})$. Since the age computed for Stock $16,5 \times 10^{6} \mathrm{yr}$, is comparable to the ages of Cen OBI and the $\mathrm{R}$ association (Herbst 1975) Cen R1 (from $10^{6}$ to $8 \times 10^{6} \mathrm{yr}$ ) and the distances of all

* Based on observations collected at the University of Toronto Southern Observatory (UTSO), Las Campanas, Chile.

$\star \star$ Table 2 is only available in electronic form at the CDS via anonymous ftp to cdsarc.u-strasbg.fr $(130.79 .128 .5)$ or via http://cdsweb.u-strasbg.fr/cgi-bin/qcat?J/A+A/430/471 $\star \star \star$ Member of the Carrera del Investigador Científico del CONICET. these groups are similar, he suggested that Stock 16 is member of a more extended and large structure. Two Wolf-Rayet stars (van der Hucht 2001), WR 50 (LSS 3013, MR 44) and WR 51 (LSS 3017, MR 45) also lie in this field, although photometric arguments (T85, van der Hucht 2001) indicate they are behind the cluster. Two other particular stars, 60a and 60b (in T85's notation), that appear embedded in a reflection nebulosity on the southeast side of the cluster at the end of the elephant trunk have been assumed to be members of a new star generation by T85. Recently, UBVRI multicolor linear polarimetry carried out by Feinstein et al. (2003), who have studied several moderately bright stars, has allowed determination of the properties of the ISM toward the cluster finding a mean polarization percentage $P \approx 2.5 \%$.

The present photometric investigation is aimed at performing a deeper study of the region including the open cluster Stock 16 and the surrounding area. We will try to confirm the earlier assumption of T85 who proposed that the star formation process in the area has taken place along the axis joining the elephant trunk structure and Stock 16. Since his assumption is mainly based on the fact that an anomalous number of very faint stars (identified by him as pre main sequence stars - PMSs - of low masses) lie on both sides (south and north) and especially to the northwest of Stock 16, a deep follow-up 


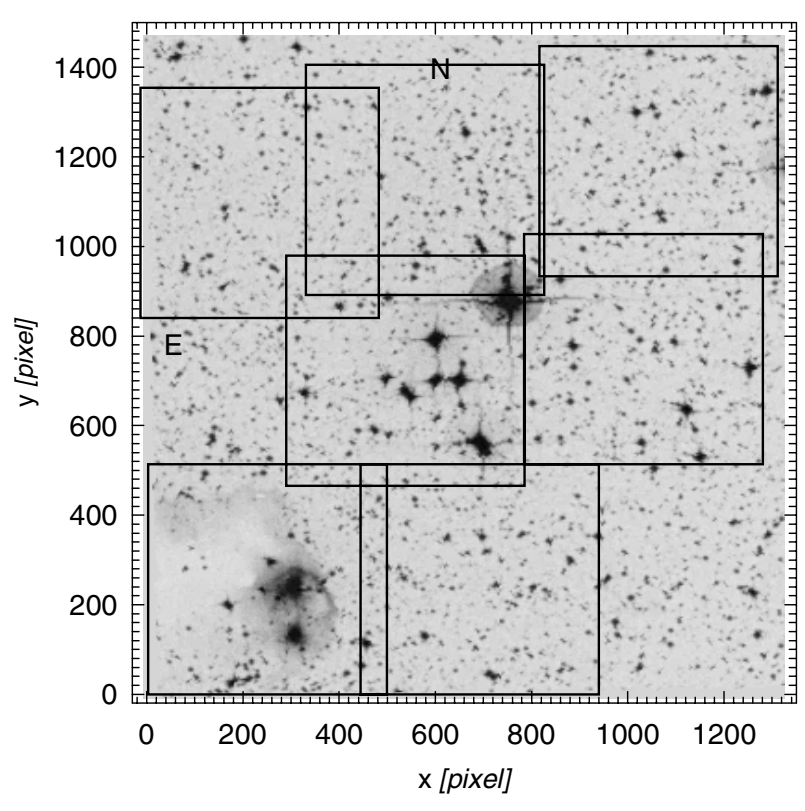

Fig. 1. The finding chart of Stock 16 adapted from a DSS chart showing the seven frames measured in the present investigation. North and East are indicated.

study will help to confirm his findings and clarify this point. Consequently, we will also attempt to reevaluate the parameters of Stock 16 and construct its initial mass function, IMF. In Sect. 2 we describe the observational procedure and the data reduction process. In Sect. 3 we discuss cluster size, membership, distance, age, the two WR stars membership and their observational properties. In Sect. 4 we compute the initial mass function of Stock 16. An overall discussion is given in Sect. 5 and the conclusions are offered in Sect. 6.

\section{Observations and data reduction}

CCD $U B V(R I)_{\mathrm{C}}$ imaging photometry was carried out in the field of Stock 16 - see the finding chart in Fig. 1 - along two observational runs at the University of Toronto Southern Observatory, Las Campanas, Chile, using the Hellen Sawyer Hogg 60-cm telescope: on the nights of 1994 April 13, 14 and 16, we obtained $U B V R I$ photometry for four frames with the nitrogen-cooled detector PM METHACROME UV coated (0.45 '/pix) covering $4^{\prime}$ on a side; three more frames were exposed on the nights of 1996 February 25 and 26 in the $U B V(I)_{\mathrm{C}}$ bands (this time, the detector was glycolrefrigerated). Exposure times ranged from 2 to $60 \mathrm{~s}$ to get photometry of the brightest stars, $1100 \mathrm{~s}$ to improve the signalnoise ratio among the faintest stars and from 100 to $200 \mathrm{~s}$ to adequately match the photometry of the brightest and faintest stars. The weather conditions at UTSO were always photometric, the seeing values ranging from $1.12^{\prime \prime}$ to $1.5^{\prime \prime}$.

Instrumental signatures of the frames were removed using bias and dome flat exposures and a test of dark current - which proved to be nonsignificant - was also done to recognize its contribution to our observations. UBVRI instrumental magnitudes were produced via the point spread function, PSF, (Stetson 1987) using DAOPHOT and the final colors and
Table 1. Errors as a function of $V$ magnitude.

\begin{tabular}{cccccc}
\hline \hline$V$ range & $\epsilon_{V}$ & $\epsilon_{(B-V)}$ & $\epsilon_{(U-B)}$ & $\epsilon_{(V-R)}$ & $\epsilon_{(V-I)}$ \\
\hline $7-15$ & 0.02 & 0.02 & 0.03 & 0.02 & 0.02 \\
$15-17$ & 0.02 & 0.04 & 0.07 & 0.03 & 0.02 \\
$17-18$ & 0.03 & 0.06 & 0.15 & 0.03 & 0.04 \\
$18-19$ & 0.03 & 0.08 & 0.20 & 0.04 & 0.04 \\
$>19$ & 0.08 & 0.25 & & 0.11 & 0.09 \\
\hline
\end{tabular}

Table 3. Differences T85 minus our CCD photometry.

\begin{tabular}{ccccccc}
\hline \hline$\Delta V$ & $\epsilon_{V}$ & $\Delta(B-V)$ & $\epsilon_{B-V}$ & $\Delta(U-B)$ & $\epsilon_{U-B}$ & $N$ \\
\hline-0.02 & 0.03 & -0.044 & 0.03 & -0.05 & 0.06 & 19 \\
\hline
\end{tabular}

magnitudes were obtained using calibration sequences in the open clusters NGC 5606 and Hogg 16 (Vázquez et al. 1991, 1994). Special care was taken that the calibration sequences include several blue and red stars to match the photometric standard system more exactly. The final errors in the calibration equations were always of the order of 0.025 or lower, and are adopted as external errors of our photometry. The internal errors, on the other hand, were computed by comparing colors and magnitudes of hundreds of stars located in the overlapping zones of our frames, the result being that, up to $V=17 \mathrm{mag}$, they are typically $<0.03$.

Despite DAOHOT errors staying low through a large $V$ range, a more realistic estimation of the uncertainty of our photometry is obtained by quadratically adding transformation errors and internal errors to the DAOPHOT ones. Their distribution per magnitude range, shown in Table 1, indicates that good $U-B$ data extend to $V=17$ and in the other color indices down to $V=19$.

Table 2 (available at CDS) lists the final photometry for 1513 stars including star identification and coordinates, $x$ and $y$. To sum up, the new information after our survey includes: 1513 stars with $B-V$ and $V, 440$ with $U-B, 1195$ with $V-R$ and 1487 with $V-I$. Additional information indicating the cross correlation with other authors' numbers and some astronomical catalogues is also provided in said table. The mean differences with the photometry quoted by T85 - where most of the stars were observed by him but others were compiled from different authors -, computed in the sense T85 minus our photometry, are shown in Table 3. Five stars shown in Table 4 were rejected in the computation of the means.

Table 3 shows systematic negative differences that can partially represent potential offsets of the present photometry and that quoted by T85. It is also true that the use of the PSF technique allows a clean removal of the star-light contribution from neighbors leading to larger magnitudes and, therefore, negative mean differences in all colors and magnitudes. On the other hand, since Shorlin et al. (2004) have pointed out that the METHACROME chip at UTSO actually generates a small non-linear Balmer discontinuity effect in the $U$ band of stars near the Balmer maximum, i.e. late B-type and A-type stars, there is a chance for part of the differences and standard 
Table 4. Stars with large differences T85 minus CCD photometry. The notation used belongs to T85.

\begin{tabular}{cccc}
\hline \hline Star & $\Delta V$ & $\Delta(B-V)$ & $\Delta(U-B)$ \\
\hline 60a & -0.36 & -0.05 & -0.01 \\
21 & -0.37 & -0.50 & -0.77 \\
22 & -0.15 & -0.04 & -0.55 \\
31 & -0.31 & 0.30 & 0.26 \\
WR 51 & -0.80 & -0.05 & -0.01 \\
\hline
\end{tabular}

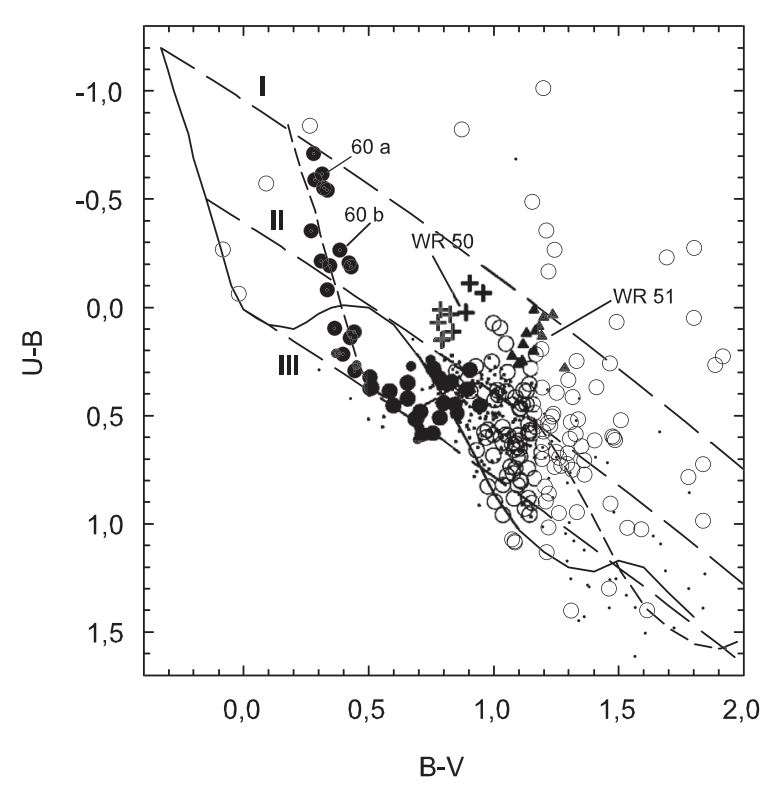

Fig. 2. The TCD of Stock 16 where members are shown as large circles. Members of Group A are indicated with big crosses and members of Group B with filled triangles. The location of the intrinsic colors (Schmidt-Kaler 1982) is shown by a continuous line and displaced by $E_{B-V}=0.51$-short dashed line. The reddening lines for O-, B5and A0-type stars labelled (I, II and III respectively). Non members of Stock 16 are shown with small filled circles. Open circles are for stars with photometric errors larger than 0.1 and dots for stars with no membership estimation. Some significant stars are pointed out.

deviations in Table 3 to obey this effect. As Shorlin et al. state, this effect is typically less than 0.05 so that no important effect that could change our conclusions is expected.

A few words are reserved for the large differences found in Table 4. We notice that star No. $21(V=13)$ is too close to star No. $1(V=7.9), 20^{\prime \prime}$ west approximately, so that the PSF technique determines its magnitude minimizing the starlight contamination due to star No. 1. Similarly, star No. 60a has a reflection nebula around it which could be successfully removed with the PSF technique as well. In the case of star No. 22 it appears blended with star No. 11 while star No. $31(V=13.8)$ has only one measure quoted by $\mathrm{T} 85$. The huge $\Delta V$ difference found for star WR 51 suggests that star light variability should not be discarded completely and that the presence of emission lines poses a clear problem for reliable photometry.

\section{Results}

\subsection{Membership}

The procedure to estimate membership and reddening in Stock 16 and the surrounding area is the same as already applied to other clusters (e.g. Vázquez et al. 1995; Baume et al. 2003) and, due to space reasons, shall not be repeated here. In the rest of this paper, all stars with errors larger than 0.1 will be shown with open circles in the respective two-color (TCD) and color-magnitude (CMD) diagrams, but the analysis will be focused only on stars with photometric errors $<0.1 \mathrm{mag}$. This limit excludes lots of faint stars but is a necessary compromise for the gain in accuracy.

The photometric diagrams shown in Figs. 2-4 (especially Fig. 2) show the presence of three main star groups, at increasing reddening values, composed of early stars. These groups are easily segregated from each other only in the TCD while they remain strongly mixed with each other and with field interlopers in the CMDs. The first of them is Stock 16 (cluster members are all shown with filled circles) which is identifiable down to $V \approx 16$ mag in the CMDs (lower and upper panels of Figs. 3) and to 0.8 in $B-V$. The other two more reddened groups are shown with big crosses and filled triangles respectively, and can be detected down to $V \approx 16-17$ mag in the CMDs.

The TCD shows a few foreground stars of spectral types later than A0. Some of them are presumably related to the foreground Cepheid star V378 Cen (marginally off the northern edge of the surveyed region, see T85). The absorption maps by Neckel \& Klare (1980) show a jump at $1.5 \mathrm{kpc}$ where the visual absorption rises to $A_{V}=1.6$ and keeps constant for the next kiloparsec. At $2.5 \mathrm{kpc}$, the absorption increases again amounting to $A_{V}=3.5$ up to $3.5 \mathrm{kpc}$. However, a pair of stars in Neckel \& Klare suggest absorption features of $A_{V}=0.5$ near the Sun at less than $0.5 \mathrm{kpc}$.

\subsubsection{The three stellar groups in detail}

We found 15 stars in Stock 16 with a unique reddening solution in the TCD. For them, individual intrinsic color and color excesses were determined in the standard way (Vázquez et al. 1995; Baume et al. 2003) obtaining the following mean values: $E_{B-V}=0.51 \pm 0.05$ and $E_{U-B}=0.36 \pm 0.04$. This mean $E_{B-V}$ is a bit larger than the previous one of 0.49 found by $\mathrm{T} 85$, probably due to a different formulation of the $E_{U-B} / E_{B-V}$ relations adopted in both papers. As this group is composed mostly of stars earlier than A0, we directly applied the relations between $(B-V)_{0},(V-R)_{0}$ and $(V-I)_{0}$ from Schmidt-Kaler (1982) and Cousins (1978) to estimate individual $E_{V-R}$ and $E_{V-I}$ color excesses. In this way we found $E_{V-R}=$ $0.26 \pm 0.04$ and $E_{V-I}=0.62 \pm 0.07$. Additionally, new cluster members have now been found; however, despite this fact and even if stars $60 \mathrm{a}$ and $60 \mathrm{~b}$ are included as potential members of Stock 16, the cluster remains poorly populated with only 27 confirmed members.

The cluster main sequence shows a sort of "gap" at $15<$ $V<16$ mag which is very noticeable in the $V / V-R$ 

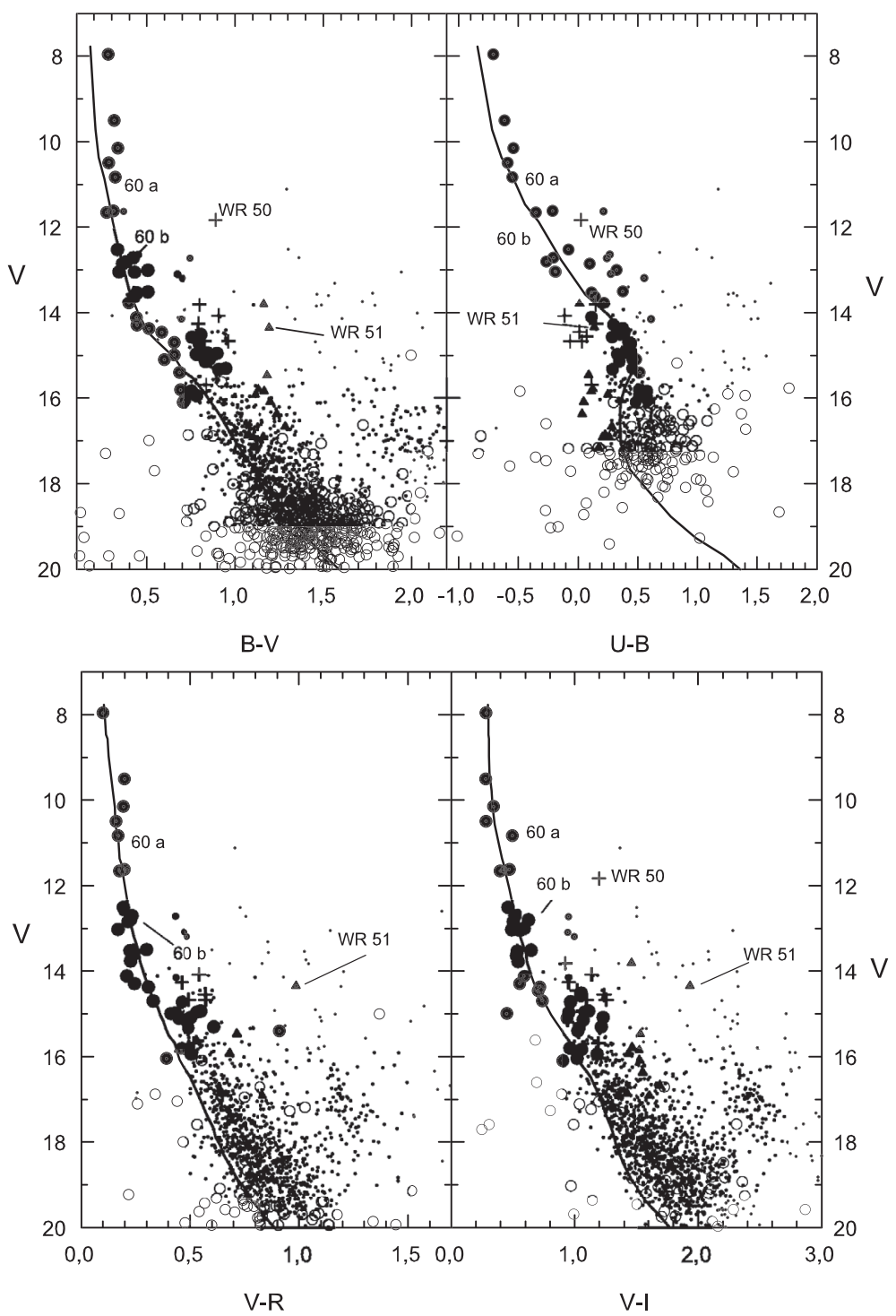

Fig. 3. The CMD diagrams of the area of the Stock 16. Upper panel: the $V / B-V($ left $)$ and $V / U-B$ (right) diagrams. Symbols as in Fig. 2. The solid lines represent the Schmidt-Kaler (1982) ZAMS fitted to an apparent distance modulus of 13.0. Lower panel: the $V / V-R($ left) and $V / V-I$ (right) diagrams. The solid line is the mean line for main sequence stars from Schmidt-Kaler (1982) and Cousins (1978) fitted to the same distance modulus as above. Symbols as in Fig. 2. In both panels some important stars are indicated.

and $V / V-I$ diagrams and belongs to the type frequently reported in the literature (see e.g., Rachford \& Canterna 2000; Giorgi et al. 2002; Carraro 2002; Tapia et al. 2003).

The second group, hereafter Group A, is composed of 9 stars that were selected from their locations between the reddening lines for $\mathrm{O}$ and $\mathrm{B} 5$ stars as shown in Fig. 2. The lower limit for B5-type stars is arbitrary and probably other stars below it are mixed with field stars in the color ranges $0.7<$ $B-V<1.5$ and $0.3<U-B<0.8$. Using the same procedure as for Stock 16 stars, the mean reddenings of Group A are $E_{B-V}=$ $0.87 \pm 0.12$ and $E_{U-B}=0.79 \pm 0.06$. Determining individual $E_{V-R}$ and $E_{V-I}$ excesses, we found mean values $E_{V-R}=$ $0.61 \pm 0.04$ and $E_{V-I}=1.34 \pm 0.1$. From the spatial point of view, members of this group are located all over the surface of our survey. Since star WR 50 (LSS 3013, V864), a WC7+OB (van der Hucht 2001; Lundström \& Stenholm 1984; T85) has reddening values similar to the typical ones of Group A, we conclude that it is part of this group and, therefore, a background star.

The third group, Group B, is defined under the same conditions as Group $\mathrm{A}$ and has the following mean color excesses (applying the procedure used above), $E_{B-V}=1.18 \pm$ $0.09, E_{U-B}=1.13 \pm 0.06, E_{V-R}=0.43 \pm 0.38$ and $E_{V-I}=$ $1.85 \pm 0.15$. The WR star, WR 51 (LSS 3017), shows the same reddening amount and is surely related to Group B. What is interesting in this group is its compact spatial distribution, northwest of Stock 16. This group and several other apparently blue stars though with dubious colors such as 77, 138, 163, 269, $301,308,427$ and 549 (our notation), are all located at $x>500$ and $y>740$, that is, northwest of Stock 16. Finally, we point out that stars Nos. 38, 146, 156, 192, 242, 308, 388 and 468 (our notation see Table 2) show an R-band deficit because their individual $E_{V-R}$ excesses are lower than the typical mean of the whole group. 


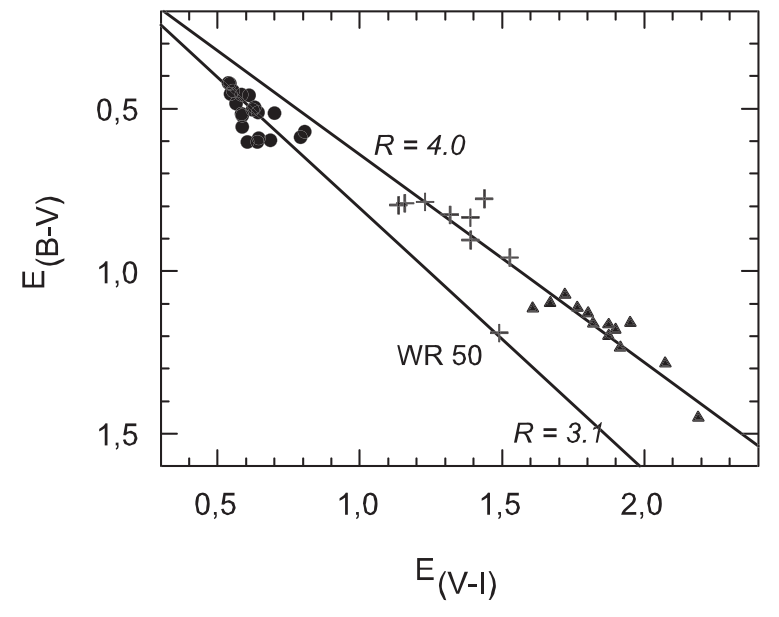

Fig. 4. The $E_{B-V}$ vs. $E_{V-I}$ diagram for Stock 16, Group A and Group B - symbols as in Fig. 2. The continuous lines show the respective data fitting for $R=3.1$ and $R=4-$ see text. The position of the W-R star WR 50 is indicated.

\subsubsection{The extinction law}

We verified the extinction law value towards the entire region. The estimation of the mean $E_{V-I} / E_{B-V}$ ratio that is closely related to the value of the total to selective absorption, that is, $R=A_{V} / E_{B-V}$, has been computed in each group. Figure 4 shows the plots of the individual excesses $E_{V-I}$ and $E_{B-V}$ for the three groups along with the lines of slopes 1.2 and 1.6 for $R=3.1$ and 4 respectively that fit each of them (notice that for a ratio of 1.24, the absorption law is assumed to be normal according to Dean et al. 1978). Except for Stock 16, the absorption law is anomalous with the following probable $R$-values:

$$
\begin{array}{ll}
E_{V-I} / E_{B-V}=1.19, R=3.1 \pm 0.1 & (\text { Stock } 16) \\
E_{V-I} / E_{B-V}=1.58, R=3.8 \pm 0.3 & (\text { Group A }) \\
E_{V-I} / E_{B-V}=1.61, R=3.9 \pm 0.1 & (\text { Group B }) .
\end{array}
$$

These $R$ values were applied to get corrected magnitudes of stars in each group. The reasons for the sudden change of the properties of the interstellar material beyond Stock 16 is beyond the scope of the present investigation; however, it is worth mentioning that such properties may change also within the same group. For example, WR 50 has $R=3.1$, although it belongs to Group A with $R=3.8$ but because of the probable presence of dust envelopes around this type of star, the determination of its $R$ value should be considered with caution.

\subsubsection{Reddening distribution across the cluster surface}

Individual $E_{B-V}$ color excesses of likely and probable members of Stock 16 and of the members of Groups A and B were used to trace the locus of equal reddening across the area investigated. We also included color excess values of obvious foreground stars with unique reddening solutions in the TCD so that in the computation of the map of Fig. 5 more than 60 stars were employed. These contour lines shown in the figure were

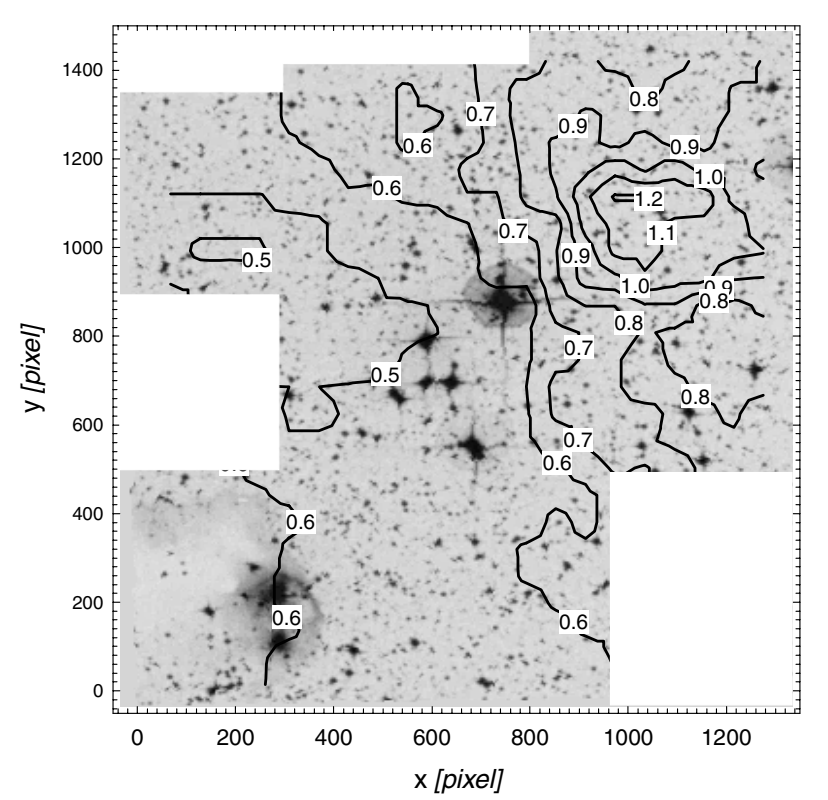

Fig. 5. The reddening contour lines over the surface of the area measured. The labels are shown in steps of $E_{B-V}=0.1$.

generated using a median filter and the contour labels were set in steps of 0.1 mag reddening.

The general reddening pattern shown in Fig. 5 varies increasingly from south-east to north-west along the axis connecting the elephant trunk with the cluster Stock 16. In the northwest corner of the figure the reddening lines appear strongly concentrated, exactly where Group B stars are found. The reddening contours also show that stars in the central part of Stock 16 are (slightly) differentially affected from southeast to north-west. That justifies what we observe in the TCD of Fig. 3, that stars 1, 5, 6, 9. 18, 60a and 60b (T85 notation) are systematically redder than the mean of the cluster by $E_{B-V}=0.1$.

\subsubsection{The distances}

Distances were obtained in the usual way by superposing the Schmidt-Kaler (1982) ZAMS onto the corrected $V_{0}$ vs. $(B-V)_{0}$ and $V_{0}$ vs. $(U-B)_{0}$ diagrams. In the case of Stock 16 stars the best fit was achieved for $V_{0}-M_{V}=11.4 \pm 0.15$ (error from eye-inspection). That implies a distance of $d=1900 \pm$ $100 \mathrm{pc}$ that confirms the earlier determination made by T85, who found 11.44.

In the case of Group A, the fitting of its members yields $V_{0}-$ $M_{V}=12.8 \pm 0.3$ (error as above) or $d=3600 \pm 400 \mathrm{pc}$, but the ZAMS fitting is not very accurate and this distance must be taken as an approximation. This way, Group A is likely to be related to the one earlier found by Jackson (1976, cited in T85), centered at $l^{\circ}=305.81$ and $b^{\circ}=1.1$ behind Stock 16 at a distance of $3600 \mathrm{pc}$ approximately.

As for Group B, the ZAMS fitting yields $V_{0}-M_{V}=$ $14.8 \pm 0.3$ (error as above) corresponding to a distance of $9000 \pm$ 1100 pc. At such a distance and located at $l^{\circ}=306.1$ and $b^{\circ}=0.2$, approximately, Group B is spatially related to a young region. In fact, an analysis of the distribution of open clusters 

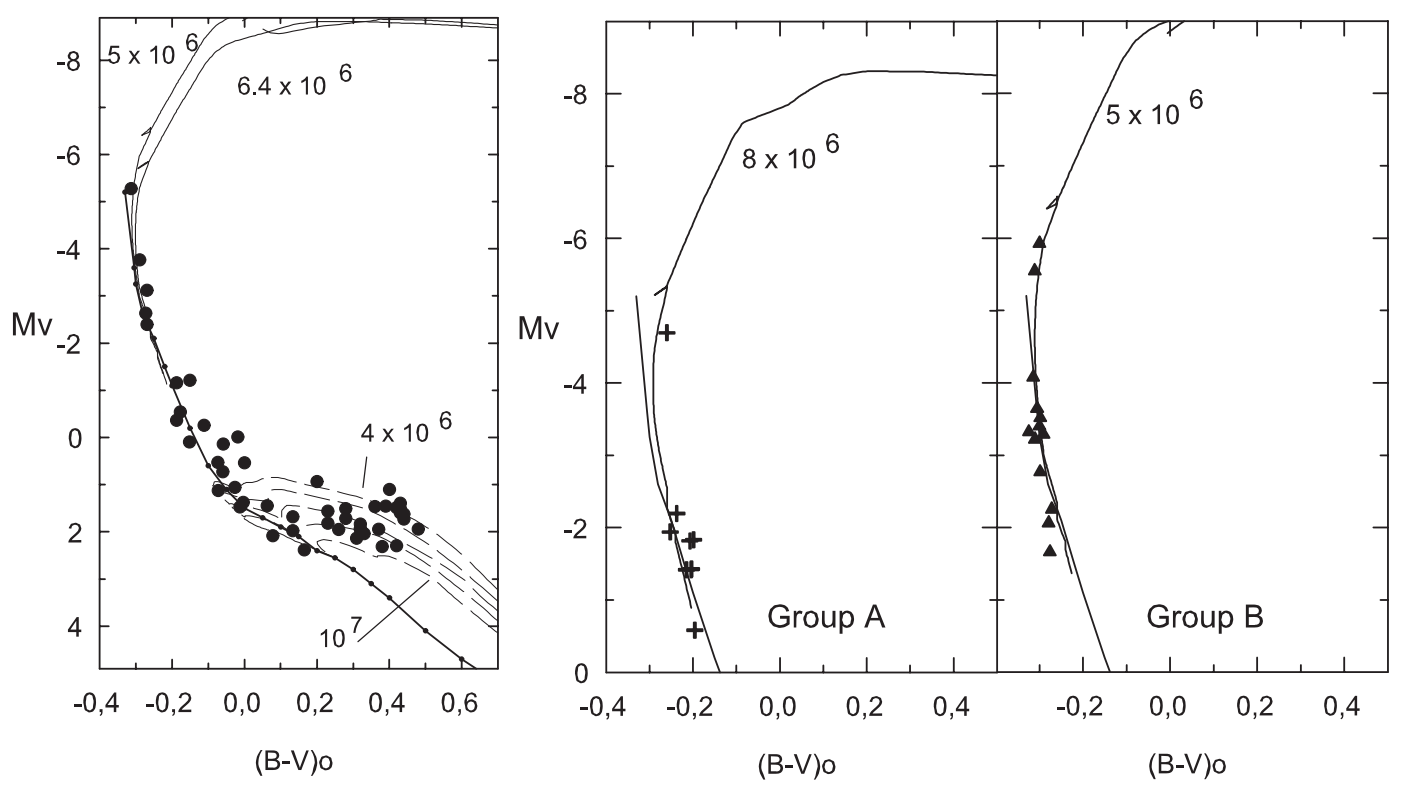

Fig. 6. The corrected CMD of the three groups. The ZAMS of Schmidt-Kaler (1982) and the isochrones of Schaller et al. (1992) - continuous lines - are fitted to each group. Numbers indicates the corresponding age. In the case of Stock 16 we show in addition the pre main sequence isochrones - dashed lines - from Bernasconi \& Maeder (1996). Symbols as in Fig. 2.

with ages < 50 Myr (taken from WEBDA) and HII regions taken from the list of Georgelin \& Georgelin (1976) shows that it is not far from the $H 109 \alpha$ regions $311.5+0.4$ and $311.6+0.3$, both at kinematic distances of $8.5 \mathrm{kpc}$ (Georgelin \& Georgelin 1976) and therefore belonging to the Scutum-Crux spiral arm.

\subsubsection{Ages}

Age estimations of the three groups were done superposing the isochrones of Schaller et al. (1992) derived from evolutionary models computed with mass loss and overshooting as shown in Fig. 6.

In the case of Stock 16, it was not possible to find a unique fitting among the evolved stars but an age range from $5 \times 10^{6} \mathrm{yr}$ to $6.4 \times 10^{6} \mathrm{yr}$, which is close to the age range $3 \times 10^{6}$ to $5 \times 10^{6} \mathrm{yr}$ earlier found by $\mathrm{T} 85$.

Figure 6 (left panel) shows also several stars of Stock 16 distributed above the ZAMS at $1.5<M_{V}<2.5$ mag showing some moderate $M_{V}$ scatter at constant color. As these stars occupy the place reserved for pre-main sequence stars PMSs (Preibisch \& Zinnecker 1999; Baume et al. 2003), an attempt to derive their ages was done superposing the pre-main sequence isochrones computed by Bernasconi \& Maeder (1996) as shown in Fig. 6 that yields they are $5 \times 10^{6}$ yr to $10^{7}$ yr old with a probable mean age of $7 \times 10^{6} \mathrm{yr}$.

A priori, as the $M_{V}$ scatter at constant color is responsible for the age spread of PMSs, the question arises whether it is reflecting a true age dispersion among these objects. It is now widely accepted that the $M_{V}$ scatter is produced by a combination of factors instead of being a real age scatter: a) a probable high number of binary stars among PMSs (see also Bessell \& Stringfellow 1999; Preibisch \& Zinnecker 1999; Hartigan et al. 1994) that widens the cluster sequence as a whole by raising the stars above a reference line such as the ZAMS; b) the random distribution of accretion disks around pre main sequence stars (Kenyon \& Hartmann 1990) that introduces an additional increase in the stellar luminosities; and c) differential reddening that may affect different stars in various ways (depending on their spectral types and spatial location). In the present case the $M_{V}$ scatter is not as important as in other young clusters probably because the internal reddening of Stock 16 is not great, so it can produce only a moderate $M_{V}$ scatter. We do not know how the other two effects are acting but given that the scatter is not so strong they probably have little effect.

By keeping in mind that the mean age of the PMSs population related to Stock 16 is not far from the mean age deduced from the upper main sequence stars it turns out to be reasonable that the star formation has been almost simultaneous. Most of the PMSs candidates are all located along the axis joining the elephant trunk with the cluster itself as shown in Fig. 7. Two of them are located around the stars $60 \mathrm{a}$ and $60 \mathrm{~b}$ and two others $4^{\prime}$ north of the elephant trunk.

The age of the other two groups is of the same order: Group A, at $3.6 \mathrm{kpc}$, is slightly older than Stock 16, with a mean age of $8 \times 10^{6} \mathrm{yr}$. That is, not too far from the age of Stock 16 and still inside the age ranges given by T85 for the nearby members of Cen OB1 and Cen R1 associations.

As for the other group, Group B, it is as young as Stock 16 with a mean age of $5 \times 10^{6} \mathrm{yr}$. So, we are dealing with a very compact star group which is not only very distant but also very young. Its proximity to the young regions indicated above and the presence of a W-R star related to it lead us to think of it as a remote OB association in the spiral arm Scutum-Crux.

\subsection{The two WR stars}

Evidence accumulated in the literature indicate that the two Wolf-Rayet stars, WR 50 and 51, located in this area, are not 


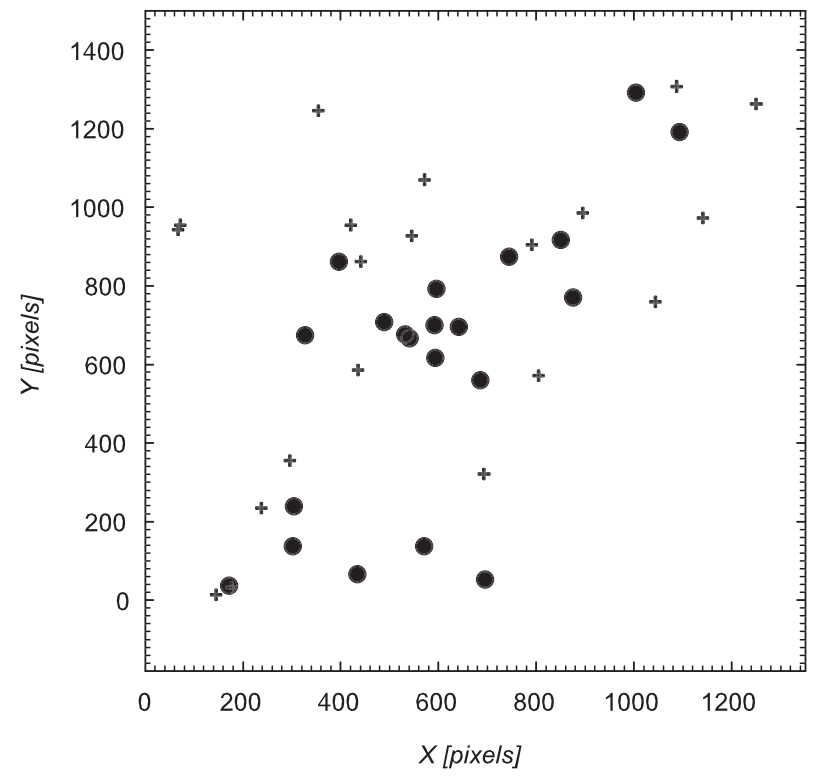

Fig. 7. The distribution of members (filled circles) and probable pre main sequence stars (crosses) in the area of Stock 16. The scale is the same used in Fig. 1.

members of Stock 16. As already said, our photometry clearly locates these two stars beyond Stock 16 and therefore, with no relation to the cluster; however, it is important to know as exactly as possible their absolute magnitudes given that they belong to the groups already described. In the following part of the study, both WR stars were de-reddened adopting $(B-V)_{0}$ intrinsic colors -0.30 and -0.26 for subtypes WC7 and WN4 respectively from Lundström \& Stenholm (1984).

\subsubsection{WR 50}

WR 50 is a variable star. van Genderen et al. (1991) estimate a $V_{J}$ mag of approximately 11.9 and have built and interpreted the light curve of this star as the result of a probable intricate eclipsing phenomenon. They also demonstrate that WR 50 shows variations in $V-B, B-L$ and $B-U$ indices. The VII catalogue of WR stars (van der Hucht 2001) includes this star among the 37 galactic binaries and probable binaries and classifies it as WC7+OB SB2 with a 1.06 (d) period (van Genderen et al. 1991). The absolute magnitude corresponding to this type of WR star, as published in the van der Hucht catalogue, should be $M_{V}=-4.5$ (quoted error bars allow up to -5 mag.). In the particular case of WR 50, the mean visual absorption cited in the catalogue amounts to $A_{V}=3.77$ while our estimation is $A_{V} \approx 4.5$ (for $R=3.1$ ), more in keeping with Morris et al. (1993) who found $A_{V}=4.44$. At the distance of Group A (where this star is assumed to belong) we find $M_{V}=-4.7$ in excellent agreement - considering the probable error in the distance modulus of Group A - with the absolute magnitude -4.97 of the system quoted by van der Hucht (2001). We already mentioned that the individual $E_{V-I} / E_{B-V}$ ratio of WR 50 is compatible with a normal absorption law characterized by $R=3.1$ what disagrees with the typical $R$-value of Group A, 3.8. However, the polarimetric value of WR 50 as indicated in Feinstein et al. (2003) $P_{V}=2.64 \%$ is in no contradiction with the distance derived here nor with its $R$ value derived above.

\subsubsection{WR 51}

This star is classified WN4+OB? in the van der Hucht (2001) catalogue; the WR companion is assumed to be an O7V star (Smith et al. 1990). van der Hucht gives $M_{V}=-5.05$ for the system while our estimation, based on the distance of the Group B yields $M_{V}=-5.9$, quite far from that value. In terms of explaining such large discrepancy we indicate that a) the visual absorption assumed by van der Hucht is $A_{V}=5.16$ while we find $A_{V}=5.6$ (for $R=3.8$ ) and $\mathrm{b}$ ) the combined magnitude of a system composed of a WN4 star with $M_{V}=-3.5$ (van der Hucht) and an $05 \mathrm{~V}$ with $M_{V}=-5$ yields $M_{V}=-5.6$, not far from our estimation. Also, the error in the distance modulus of group B is of the order of $0.3 \mathrm{mag}$ and is surely contributing to the large difference. The apparent magnitude quoted by $\mathrm{T} 85$ for this star is $V=13.55$, but our photometry gives $V=14.34$ (0.8 mag. fainter). Does this also indicate a strong variability of the system not reported anywhere as far as we know? WR 51 shows, on the other hand, the highest polarization value, 4.77\%, as given in Feinsten et al. (2003), which is congruent with its large distance.

\section{The initial mass function of Stock 16}

The initial mass function, IMF, is defined as the number of stars per mass interval assuming they formed at the same time and in the same region of space. To build the IMF of Stock 16 we superposed evolutionary tracks of Schaller et al. (1992) onto the cluster members as shown in Fig. 8 with continuous lines. Also, with short dashed lines we show the pre-main sequence evolutionary tracks derived by Bernasconi \& Maeder (1996). The stellar masses of cluster members were estimated as follows: for stars in the H-burning phase - stars with masses larger than $3 M_{\odot}$ - the individual ZAMS masses were obtained by redrawing the individual path of each star back to their initial location in the ZAMS; for PMS stars - from 2.5 to $1.5 M_{\odot}-$ ZAMS masses were all obtained by reconstructing the individual path of each star up to their final location in the ZAMS.

The number of stars in the ZAMS found between two consecutive evolutionary tracks is given in Table 5. Notice that arbitrary mass bin sizes were used to avoid empty bins and each of them was assigned a mean stellar mass (the central one). To scale the values, the number of stars in each bin was divided into the $\Delta \log M$.

So as to get the slope of the frequency distribution, if $\mathrm{N}$ is the number of stars in a bin with central mass $\mathcal{M}$, then the value of the slope $x$ of the mass distribution is determined by:

$x=-\frac{\log (\mathrm{d} N / \Delta \log \mathcal{M})}{\log \mathcal{M}}$.

The mass points of Stock 16 are shown in Fig. 9 together with the line showing the un-weighted fitting from a least squares method. To minimize incompleteness effects, the less massive mass point was ignored in the fitting. The fitting line has a 


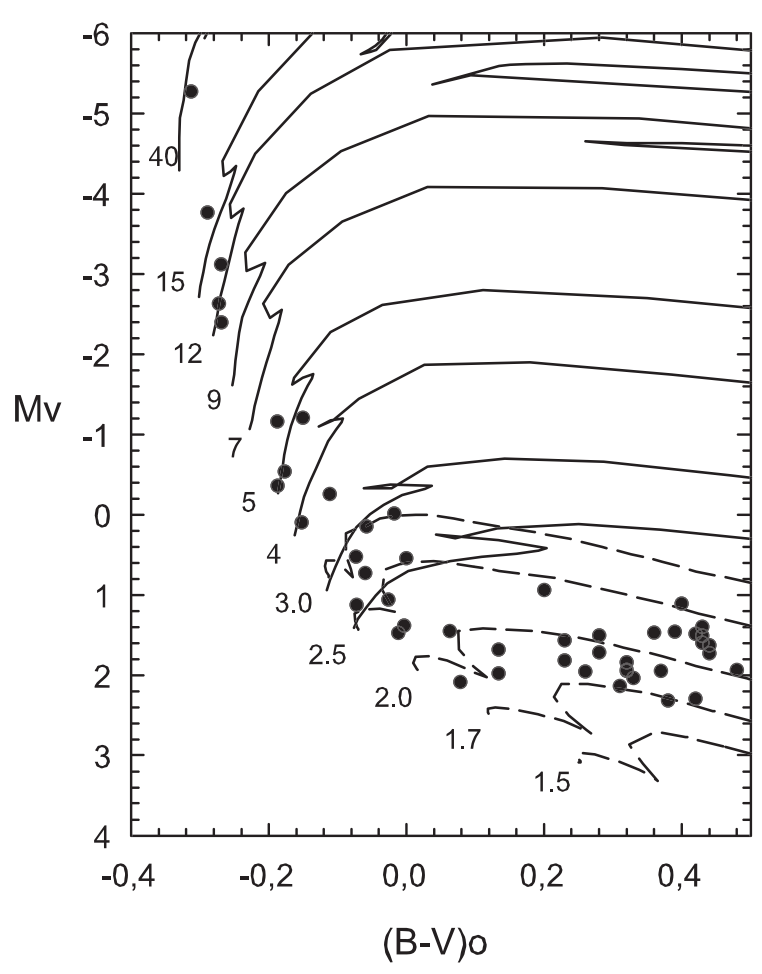

Fig. 8. The evolutionary tracks of Schaller et al. (1992) - continuous lines - and Bernasconi \& Maeder (1996) - dashed lines - for post main sequence and pre main sequence stages respectively. The stellar mass is indicated. Filled circles denote members of Stock 16.

Table 5. The initial mass function of Stock 16.

\begin{tabular}{cccc}
\hline \hline$\langle M\rangle$ & $N$ & $\log (M)$ & $\log \frac{\mathrm{d} N}{\Delta \log (M)}$ \\
\hline 1,6 & 4 & 0,20 & 1,8 \\
1,8 & 14 & 0,25 & 2,0 \\
2,2 & 14 & 0,34 & 2,2 \\
2,7 & 6 & 0,43 & 1,9 \\
3,4 & 4 & 0,53 & 1,5 \\
4,4 & 3 & 0,64 & 1,3 \\
7,5 & 2 & 0,87 & 0,7 \\
13,2 & 2 & 1,12 & 1,3 \\
24 & 2 & 1.38 & 0.7 \\
\hline
\end{tabular}

slope $x=-1.3 \pm 0.4$ that in principle (considering the quoted errors in Fig. 9, estimated as the $\sqrt{N}$ of the counts - vertical bars - and the fitting errors) coincides with a typical Salpeter (1955) slope of 1.35 .

Apart from the formal errors of the least squares fitting, the IMF slope we have found here is subject to some unavoidable uncertainties such as unresolved binaries, stellar evolution and membership uncertainties. The case of binaries is probably the most important and difficult to estimate: if the number of binaries in the cluster is high, it produces a slope value flatter than it actually is (Richtler \& Sagar 1991). Kroupa et al. (1991, 1992) also dealt with this point and reached the conclusion that if a Salpeter model is assumed to be valid, then the results may lead to an apparent deficiency in low mass

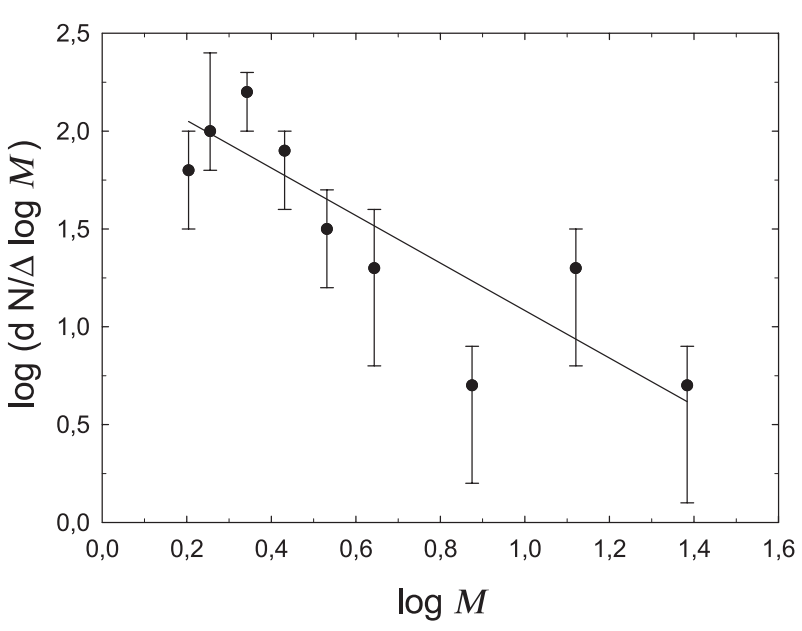

Fig. 9. The mass points of Stock 16 fitted with a least squares method - continuous line. Vertical bars are the $\sqrt{N}$ of the counts.

stars and, therefore, the slope of very young but distant open clusters would become flatter too. Binary stars do not seem to be an important source of uncertainty in the IMF slope computation as we do not find evidence for a large number of them in the Stock 16 upper main sequence. Just a few cluster stars rise above the ZAMS moving simultaneously redwards as binaries do. Finally, when comparing the slope of Stock 16 obtained in this work with those quoted by Sagar et al. (2001) we see that it fits well with galactocentric, galactic longitude and age dependencies shown in their Fig. 22.

\section{Discussion}

Stock 16 is a young cluster located at the end of the elephant trunk emerging on the south west side of a prominent dust concentration. In agreement with T85, we also see that the cluster seems to have formed in the outermost edge of the primitive elephant trunk. T85 found an anomalous high star density round the cluster and called attention to an unusual peak of star density $2^{\prime}$ northwest of star No. 1 (T85 notation) that he identified with the former leading edge of the elephant trunk. We investigated the star density at increasing $V$ magnitudes across a larger area of $20^{\prime} \times 20^{\prime}$ using a DSS plate (first generation) centered in Stock 16. Magnitudes of stars in the plate were obtained using DAOPHOT - aperture photometry - and transformed to the CCD system using a number of our own stars with CCD measures. The plate limit was found close to $V=$ 17.5. Examining the spatial distribution of all the stars detected above a given threshold we found only a secondary (and significant) peak of star density "southeast" of the elephant trunk (apart from the one associated with the cluster itself), that we relate to the infrared stellar group $\mathrm{Stk} 16 \mathrm{se}^{\mathrm{c}}$ spatially associated with the the reflection nebula RN60a according to Dutra et al. (2003). No other obvious density peak was found.

So, we find that T85's finding that the anomalous high star density round the cluster is a clue of low mass star formation activity is essentially right despite finding no abnormal star density. We have identified some probable PMSs in the area but not at the level of relating them to a star density peak $2^{\prime}$ min northwest of star No. 1. Indeed, there is a density peak but it 
is only a marginal one composed of blue faint stars that we associated with a distant $\mathrm{OB}$ association at $9 \mathrm{kpc}$ approximately, in the Scutum-Crux arm, and not the result of an accumulation of PMSs.

The analysis of our data indicates that Group A with a mean reddening $E_{B-V}=0.87$ is spatially related to the anonymous background association indicated by Jackson (1976) and that between Stock 16 and Group A, the space is devoid of dust as suggested by T85. It is interesting that the age of Group A is similar to the one of Cen OB1 as it has been argued by T85 that a sort of sequential star formation has taken place in the whole area where Stock 16 is not necessarily the last star generation but a new star generation is now in progress. Probably, some residual activity of such a process could be the presence of the infrared stellar group Stk16se ${ }^{c}$ at the east border of the dust cloud south of the elephant trunk. Of course, its nature should be confirmed, but a few of our PMSs candidates are seen projected against this latter group. On the other end, northwest of Stock 16, the IRAS source IRAS 13147-6210 is found at $\alpha_{2000}=13: 18: 03.4$ and $\delta_{2000}=-62: 26: 46$, very close to WR $50\left(\alpha_{2000}=13: 18: 01\right.$ and $\left.\delta_{2000}=-62: 26: 04\right)$ which may be a hint of late star formation activity. In the cluster itself, the process of star formation is still active as several PMS candidates with masses from 3 to $1.5 M_{\odot}$ have been found. The fact that the mean ages of both H-burning stars and PMSs candidates are similar $\left(6-7 \times 10^{6} \mathrm{yr}\right)$ allows us to suggest that the star formation process in the area was simultaneous, consistent with our recent finding in another young open cluster, NGC 3293 (Baume et al. 2003) and took place within a few million years, confirming, to some extent, T85's earlier assertion that new stars are born in the area.

As found in other clusters (e.g. NGC 6231 and NGC 3293, Baume et al. 2001, 2003 respectively), Stock 16 shows a sharp bend at the magnitude bin $M_{V}=+1 \ldots+2.5 \mathrm{mag}$. Indeed, several stars located at this bend belong to the PMSs population as shown in Fig. 8. The bend in Stock 16 is accompanied by a paucity in the star number found at the apparent magnitude range $14<V<16$ (obvious in the $V / V-R$ and $V / V-I$ diagrams) that we interpret as the settlement of a gap of the type observed in the Carina cluster Trumpler 16 (Tapia et al. 2003) among others. Unfortunately, the lower mass limit of the gap is hidden by field stars and we cannot estimate its magnitude nor do we think it probable that this feature is a random fluctuation of the star number along the cluster sequence (Giorgi et al. 2002; Carraro 2002), but it should be very interesting to confirm its true nature.

As for the cluster IMF slope value, given the formal errors in applying a least squares method, the value of $x=1.3 \pm 0.4$ is not different from the typical value of 1.35 derived by Salpeter (1955). However, Scalo (1998) has questioned the procedure of comparing IMFs derived in open clusters with the ones derived from field stars, as they come from a mixture of different populations under different evolutionary conditions, environments and distances. Finally, the determination of the IMF slope $x$ is subject to so many uncertainties (part of which were described above) that searching for an universal value may be useless. In the present case we merely affirm that the IMF slope of Stock 16 is inside the range of variation described by
Scalo (1998) and certainly close to the values $-1.7 \pm$ 0.5 to $-1.3 \pm 0.5$, which are valid for the mass range from $1-100 M_{\odot}$.

2MASS data were also employed in our analysis. $V$ vs. $V-$ $K$ and $J-H$ vs. $H-K$ diagrams were constructed using 571 stars with infrared excesses and errors lower than 0.1 . These data confirm all our optical analysis, including the fact that the extinction law for Stock 16 is certainly normal as cluster stars are all distributed along the galactic reddening vector in the $J-H$ vs. $H-K$ diagram as given by Koornneef (1983). Unfortunately, few stars included in groups A and B were detected and, moreover, they are too faint to trust their IR colors and use them to study the absorption law towards these two groups. It is interesting that WR 50, in particular, shows an $R$ value close to 3 but, as demonstrated by van Genderen (1991), the photometric properties of this star are also changing with time so that it is difficult to assess the reasons for showing a normal $R$ value in a region with interstellar medium of anomalous properties.

WR 50 and WR 51 are conspicuous members of Groups A and $\mathrm{B}$, respectively. In this respect, when comparing our findings with those from van der Hucht (2001) we found that: a) for WR 50 van der Hucht gives a distance of $d=5.5 \mathrm{kpc}$ farther out than our estimation that places this star at $3.6 \pm 0.4 \mathrm{kpc}$. We place this star closer than van der Hucht because we computed a larger absorption and used an $R$ value which is not normal. As for WR 51, van der Hucht sets this star at $8.1 \mathrm{kpc}$, a distance consistent with our estimation of $9 \pm 1 \mathrm{kpc}$ assuming it is a member of a distant association in Scutum-Crux and taking into account the errors in the distance modulus of this group. It remains an open question whether this star is also variable, as our $V$ magnitude does not coincide with the magnitude quoted by $\mathrm{T} 85$.

Finally, we found a handful of young stars composing Group B at approximately $9 \mathrm{kpc}$ in the Scutum-Crux spiral arm. While the TCD demonstrates clearly its existence, confirmation by spectroscopy is clearly needed.

\section{Conclusions}

New members of the young open cluster Stock 16 have been found in the present investigation and some other stars were found in the field as candidates to become PMS stars. The mean ages of both H-burning stars and PMS stars are simi$\operatorname{lar}\left(6-7 \times 10^{6} \mathrm{yr}\right.$ approximately) so that we are in a position to affirm that the star formation process in the area has been simultaneous. On the contrary, we cannot state firmly that the number of binary stars in Stock 16 is large; our photometry does not suggest this. The cluster IMF is a typical one following the Scalo (1998) caveats.

Immediately behind Stock 16 and, therefore Cen OB1, there appears another sparse group of early stars which is likely associated with a background anonymous association. The age of this group is $8 \times 10^{6} \mathrm{yr}$.

A third star group belongs to a remote OB association at more than $9 \mathrm{kpc}$, located at the Scutum-Crux spiral arm. This is also a very young stellar group with an age of about $5 \times$ $10^{6} \mathrm{yr}$ close to or immersed in a region of recent star formation. 
Other probable members of this group are seen in our diagrams but their magnitude and colors are too unsure to extract any conclusions from them.

This article is partially based in the Digitized Sky Survey that was produced at the Space Telescope Science Institute under US government grant NAGW-2166. Original plate material is copyright the Royal Observatory Edinburgh and the Anglo-Australian Observatory

This research has made use of the SIMBAD database, operated at CDS, Strasbourg, France and of WEBDA, an open cluster data base developed by Jean-Claude Mermilliod

Acknowledgements. The authors warmly acknowledge constructive suggestions of our careful referee Dr. D. G. Turner that greatly contributed to improve this article. Thanks are also due to K. A. van der Hucht for the critical comments on the analysis of the data of the two WR stars. The authors also acknowledge the financial support from the Facultad de Ciencias Astronómicas y Geofísicas UNLP - and the IALP-CONICET. We are, once again, indebted to Bob Garrison for the allocation of telescope time at the UTSO.

\section{References}

Baume, G., Vázquez, R. A., Carraro, G., \& Feinstein, A. 2003, A\&A, 402, 549

Bernasconi, P. A., \& Maeder, A. 1996, A\&A, 307, 829

Bessell, M. S., \& Stringfellow, G. S. 1993, ARA\&A, 31, 433

Carraro, G. 2002, MNRAS, 331, 785

Cousins, A. W. J. 1978, MNSSA, 37, 62

Crampton, D. 1971, AJ, 76, 260

Dean, J. F., Warren, P. R., \& Cousins, A. W. J. 1978, MNRAS, 183, 569

Dutra, C. M., Bica, E., Soares, J., \& Barbuy, B. 2003, A\&A, 400, 533

Feinstein, C., Baume, G. L., Vergne, M. M., \& Vázquez, R. A. 2003, A\&A, 409, 933

Georgelin, Y. M., \& Georgelin, Y. P. 1976, A\&A, 49, 57

Giorgi, E. E., Vázquez, R. A., Seggewiss, W., \& Baume, G. L. 2002, A\&A, 381, 884

Herbst, W. 1975, AJ, 80, 212
Hartigan, P., Strom, K. M., \& Strom, S. E. 1994, ApJ, 427, 961

Kenyon, S. J., \& Hartmann, L. W. 1990, ApJ, 349, 197

Koornnef, J. 1983, A\&A, 128, 84

Kroupa, P., Tout, C. A., \& Gilmore, G. 1991, MNRAS, 251, 293

Kroupa, P., Gilmore, G., \& Tout, C. A. 1992, AJ, 103, 1602

Lundström, I., \& Stenholm, B. 1984, A\&AS, 58, 163

Lyngå, G. 1970, in The Spiral Structure of Our Galaxy, ed. W. Becker, \& G. Contopoulos (Dordrecht: Reidel), IAU Symp., 38, 270

Morris, P. W., Brownsberger, K. R., Conti, P. S., Massey, P., \& Vacca, W. D. 1993, ApJ, 412, 324

Neckel, Th., \& Klare, G. 1980, A\&AS, 42, 251

Preibisch, T., \& Zinnecker, H. 1999, AJ, 117, 2381

Rachford, B. L., \& Canterna, R. 2000, AJ, 119, 1296

Sagar, R., \& Richtler, T. 1991, A\&A, 250, 334

Sagar, R., Munari, U., \& de Boer, K. 2001, MNRAS, 327, 23

Salpeter, E. E. 1955, ApJ, 121, 161

Scalo, J. 1986, Fundam. Cosmic Phys., 11, 1

Scalo, J. 1998, in The Stellar Initial Mass Function, 38th Herstmonceux Conf., ed. G. Gilmore, \& D. Howell, ASP Conf. Ser., 142, 201

Schaller, G., Schaerer, D., Meynet, G., \& Maeder, A. 1992, A\&AS, 96, 269

Schmidt-Kaler, Th. 1982, in Landolt/Bornstein, New Series Group VI, Vol. 2

Shorlin, S. L., Turner, D. G., \& Pedreros, M. H. 2004, PASP, 116, 170

Stetson, P. B. 1987, PASP, 99, 91

Tapia, M., Roth, M., Vázquez, R. A., \& Feinstein, A. 2003, MNRAS, 339, 34

Turner, D. G. 1985, ApJ, 292, 148

van der Hucht, K. A. 2001, The VIIth Catalogue of Galactic Wolf-Rayet Stars, New Astron. Rev., 45, 135

van Genderen, A. M., et al. 1991, in Wolf-Rayet Stars and Interrelation with other Massive Stars in Galaxies, ed. K. A. van der Hucht, \& B. Hidayat, IAU Symp., 143, 129

Vázquez, R. A., \& Feinstein, A. 1991, A\&AS, 90, 317

Vázquez, R. A., Baume, G. L., Feinstein, A., \& Prado, P. 1994, A\&AS, 106,339

Vázquez, R. A., Will, J.-M., Prado, P., \& Feinstein, A. 1995, A\&AS, 111,85

Walborn, N. R. 1973, AJ, 78, 1067 